\title{
Performance Analysis of Joint Radio Resource Management Strategies for Beyond 3G Networks
}

\author{
Hermes Irineu Del Monego ${ }^{\# 1}$, José Manuel Oliveira ${ }^{* 2}$, Manuel Ricardo ${ }^{\# 3}$ \\ \# INESC Porto, Faculdade de Engenharia, Universidade do Porto \\ * INESC Porto, Faculdade de Economia, Universidade do Porto \\ Rua Dr. Roberto Frias, 378, 4200-465, Porto, Portugal \\ ${ }^{1}$ hmonegodinescporto.pt \\ 2 jmodinescporto.pt \\ ${ }^{3}$ mricardodinescporto.pt
}

\begin{abstract}
In beyond $3 G$ networks the user will not be aware of the access network technology used to provide a telecommunications service. Heterogeneous network technologies will be seamlessly integrated in one "common" access network, enabling users to move around and continuously receive their subscribed services. In a commercial environment, this network evolution requires that a telecommunications operator jointly manages its networks resources to improve the service offered to the users and, at the same time, to increase its revenue.

Starting from the UMTS and WLAN interconnection architecture defined by 3GPP, this paper analyzes the performance of a new joint radio resource management strategy, comparing it with two well-known strategies used in scenarios where both networks, the UMTS and the WLAN, are interconnected. The new strategy presented in the paper bases its decisions on criteria related to user mobility characteristics and the application characteristics. The strategy also introduces the possibility of renegotiating new calls and reallocating running calls from one access network to another.

The performance analysis considers two traffic scenarios. One where only real-time applications are running and other which also introduces TCP applications. The comparison studies show the proposed strategy outperforms the other strategies in what concerns call blocking probability and applications QoS support. Besides, the proposed strategy tends to reduce the handoffs between networks.
\end{abstract}

Index Terms-Renegotiation, Reallocation, Resource management, Heterogeneous wireless networks, Session continuity

\section{INTRODUCTION}

The recent evolutions in telecommunications have been influenced by the crescent need of users to access their subscribed services in mobile environments. This demand has determined two complementary research lines in this area. On one hand, multimode terminals have been developed, being capable to access different network technologies, particularly wireless technologies, such as Universal Mobile Telecommunication System (UMTS), Wireless Local Area Network (WLAN), or Bluetooth. On the other hand, the interconnection of different access networks has been researched and defined, enabling call transference from one interface to another, seamlessly to the user.

In a context where one telecommunications operator administrates different network technologies in the same physical location, a joint and efficient management of those networks resources is desired. This will certainly improve the service offered to the users and, at the same time, will increase the operators' revenue.

The $3^{\text {rd }}$ Generation Partnership Project (3GPP) has defined a generic interconnection architecture between $3 \mathrm{G}$ networks and WLAN, illustrated in Fig. 1 [1], [2]. The release 8 of 3GPP promotes solutions for networks interconnection following a seamless mobility approach, characterized by providing the service independently of the technology used to access it.

Considering the UMTS and WLAN networks interconnection scenario defined by $3 \mathrm{GPP}$, this paper analyzes the performance of a new joint radio resource management (JRRM) strategy, comparing it with two well-known strategies used in scenarios where the user is located in a place covered by different radio access technologies. This new strategy, called MTend, bases its behavior in criteria related with user mobility. Additionally, in a scenario of low available resources, the strategy introduces the possibility of renegotiating requests of new calls or reallocating existing calls from one network to another. The performance analysis presented in this paper only considers scenarios where the user is static.

In the next section, some important related works are presented. Section III presents the call admission control mechanisms used by the UMTS and WLAN interfaces. Section IV proposes the new strategy for joint management of multi-radio resources, based on user mobility and the renegotiation or reallocation of calls; this section also describes the decision algorithm used to define the interface to which a new call should be allocated. Section V presents the simulation scenario used to analyze and compare the performance of this new joint radio resource management strategy with two other wellknown strategies. Section VI analyzes in detail the simulation results. Finally, Section VII presents the conclusions and points out directions for future work.

\section{RELATED WORK}

The joint multi-radio resource management is an well accepted research area in telecommunications. It promotes solutions for the support of Quality of Service (QoS) classes while optimizing the usage of resources. 


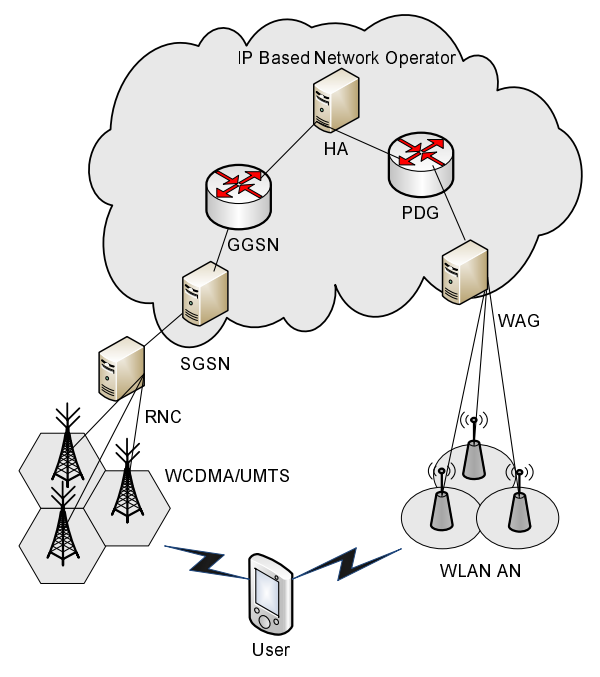

Fig. 1. 3GPP generic architecture for the interconnection of 3G and WLAN networks

A strategy based on service classes is proposed in [3], where the authors use two policies: the first policy gives priority to voice calls in GERAN and interactive traffic in UTRAN; the second inverts the choice. According to the authors, when the mobile stations are close to the cell there is no difference between both strategies. On the other side, when the distance to the cell increases, more transmission errors may occur due to WCDMA characteristics, leading to a higher degradation in UTRAN users. In this case, the first policy is the most efficient one.

Two other strategies commonly used in this context are the load balancing (LBal) and the coverage area (CovAr) strategies, which will be used for comparison with the strategy proposed in this paper. The LBal strategy is characterized by directing calls to the interface with lowest load, maintaining the interfaces balanced [3], [4], [5].

The CovAr strategy directs calls preferentially to the interface associated to smaller area cell. For example, in hotspots the calls are directed firstly to the WLAN network until it saturates, and then to the UMTS network [4].

The use of JRRM techniques to cope with multi-RAT and multi-hop networks of the same or different administrative entities, in the context of the Ambient Networks concept, is covered in [?], [?]. The presented solution is characterized by being scalable, flexible and applicable to Personal Area Networks (PANs) and Body Area Networks (BANs), as well as to large operator networks.

\section{Single Interface CAll Admission Control}

The following two sections present the call admission control mechanisms used by the UMTS and WLAN network technologies for managing new calls.

\section{A. UMTS Interface}

In UMTS, the load factor estimates the amount of supported traffic per base station site; in [6], the authors describe the mechanism to obtain the available UMTS load factor for both link directions. The load factor for the uplink direction is given by:

$$
\eta_{U L}=(1+i) \cdot \sum_{j=1}^{N} \frac{1}{1+\frac{W}{\left(E_{b} / N_{0}\right)_{j} \cdot R_{j} \cdot v_{j}}},
$$

where $N$ is the number of stations, $v_{j}$ is the activity factor of station $j$ at physical layer, $E_{b} / N_{0}$ is the signal-to-noise ratio, $W$ is the chip rate, $R_{j}$ is the bit rate of station $j$ and $i$ is the intercell interference observed by station $j$.

The load factor for the downlink direction is given by:

$$
\eta_{D L}=\sum_{j=1}^{N} v_{j} \frac{\left(E_{b} / N_{0}\right)_{j}}{W / R_{j}} \cdot\left[\left(1-\alpha_{j}\right)+i_{j}\right],
$$

where $\alpha_{j}$ is the channel orthogonality of the station $j$, while $i_{j}$ is the signal of other cells received by station $j$. For $v_{j}, \alpha_{j}$ and $i_{j}$ we used commonly accepted values proposed in [6].

The load factor is controlled to be always below a limit represented by $\eta_{\max }\left(\eta_{\max }<1\right)$. In most of the UMTS systems, the $\eta_{\max }$ value is not higher than 0.75 , for both directions, the uplink and the downlink [6], [7]. In our work, we assumed that the operator applies this value for $\eta_{\max }$ in both direction.

In the UMTS interface, a new call specifies the minimum QoS requirements that should be satisfied by the network. This request includes values for different QoS parameters, such as the bit rate. A new call is accepted by the call admission control mechanism, either in the uplink or in the downlink, when the load factor is below $\eta_{\max }$ after integrating the new call.

\section{B. WLAN Interface}

Comparing with UMTS, the WLAN interface usually offers a higher bandwidth and a smaller cell coverage area. On the other hand, the radio access in WLAN is most of the times uncertain, being based on the transmission silence time [8].

The strategy used for obtaining the available bandwidth in the WLAN interface consists on monitoring the channel occupation ratio, which determines the channel capacity for transmit [9]. New calls are only admitted in WLAN when the requested bandwidth is below the available bandwidth. The WLAN available bandwidth, in bit/s, is given by:

$$
B w_{W L A N}=\frac{L \cdot\left(R_{t h}-R_{o}\right)}{T},
$$

where $L$ is the average packet size, $T$ is the average time for having success in transmission and $R_{t h}$ is the network threshold, which is a parameter controlled by the operator [9].

The network occupation factor is given by:

$$
R_{o_{i}}=\alpha \cdot \frac{t_{\text {busy }}}{\Delta_{t}}+(1-\alpha) \cdot\left(R_{o_{i-1}}\right),
$$

where $t_{\text {busy }}$ is the busy state time, $\Delta_{t}$ is the window size measurement time, and $\alpha$ is the importance of the current sample $i$. 
When the RTS/CTS mechanism is not used, $T$ is given by:

$$
T=D I F S+T[E P]+S I F S+A C K .
$$

On the other hand, when the RTS/CTS mechanism is used, $T$ is given by:

$T=D I F S+R T S+S I F S+C T S+S I F S+T[E P]+S I F S+A C K$

where $T[E P]$ is the transmission time for a packet with payload size of EP and RTS,CTS and $A C K$ are the transmission times of $R T S, C T S$ and $A C K$, respectively.

\section{JRRM BASED ON USER MOBILITY, AND CALL RENEGOTIATION AND REALLOCATION}

The multi-radio resource management strategy proposed in this paper, called MTend, aims to maximize the use of the radio resources, while satisfying the QoS requirements posed by the applications.

Fig. 2 represents the structure of the decision algorithm used for the joint management of interconnected UMTS and WLAN radio resources under the control of the same operator. When a new call request arrives, the algorithm decides to which interface it should be directed, based on the call characteristics and resources available. In a scenario where both networks have resources available, the strategy is based on the Mobility Tendency of the users, differentiating applications according to their tendency for mobility. For example, voice calls are inherently mobile, since the probability of a user receiving or starting a call in movement is relatively high. Thus, this strategy gives priority to mobile applications in the UMTS network, in order to avoid vertical handoffs between different network technologies. Applications usually used in static contexts (e.g., web browsing and videostreaming) are accepted preferentially in the WLAN network.

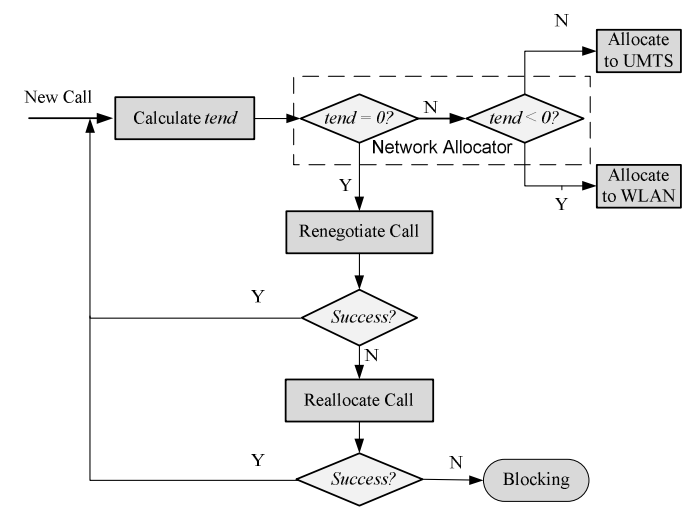

Fig. 2. Joint multi-radio resource management algorithm

In scenarios of insufficient resources, our strategy proposes two complementary mechanisms. The first mechanism consists of renegotiating the resources requested by the new call; the second mechanism considers the possibility of reallocating an accepted call from one network to the other, enabling resources to be freed in the congestioned network. The renegotiation and reallocation mechanisms require the monitoring of each access network so that their level of congestion can be evaluated.

The renegotiation mechanism consists of two renegotiation tries. In a case where the first try does not succeed, the system enables the application to reduce again the resources requested. Table I contains the renegotiation parameters for the applications used in our study.

TABLE I

APPLICATIONS BIT RATES AND THEIR RENEGOTIATION ALTERNATIVES

\begin{tabular}{|c|c|c|c|}
\hline \multirow{2}{*}{ Application } & \multicolumn{3}{|c|}{ Mean bit rate (kbit/s) } \\
\cline { 2 - 4 } & $1^{\text {st }}$ Request & $1^{\text {st }}$ Renegotiation & $2^{\text {nd }}$ Renegotiation \\
\hline Voice & 24 & 12 & 8 \\
\hline Videostreaming & 128 & 64 & 32 \\
\hline www & 128 & 64 & 32 \\
\hline FTP & 128 & 64 & 32 \\
\hline
\end{tabular}

The following paragraphs formalize the MTend strategy decision algorithm. The network interface selected to serve the new call is chosen according to Eq. (7), where tend represents the decision tendency. The indicator function $1_{y}$ is 1 if the event $y$ is true, else it is zero. $P_{U M T S}$ and $P_{W L A N}$ are the eligibility degrees given to an arriving session, respectively to be transported over the UMTS and WLAN interfaces, as show in Table II.

tend $=P_{U M T S} * \mathbf{1}_{\eta a_{U M T S} \geq \Delta_{\eta r}}-P_{W L A N} * \mathbf{1}_{B w_{W L A N} \geq r}$

TABLE II

UMTS AND WLAN ELIGIBILITY DEGREES ACCORDING TO THE USER MOBILITY CRITERION

\begin{tabular}{|c|c|c|}
\hline \multirow{2}{*}{ Application } & \multicolumn{2}{|c|}{ Eligibility Degree } \\
\cline { 2 - 3 } & $P_{U M T S}$ & $P_{W L A N}$ \\
\hline Voice & 2 & 1 \\
\hline Videostreaming & 1 & 2 \\
\hline WWW & 1 & 2 \\
\hline FTP & 1 & 2 \\
\hline
\end{tabular}

The $\eta a_{U M T S}$ and $B w_{W L A N}$ variables are, respectively, the available UMTS load factor and the available bandwidth in the WLAN interface. $\eta a_{U M T S}$ is the difference between $\eta_{\max }$ and $\eta_{D L}$, which is given by Eq. 2 , while $B w_{W L A N}$ is given by Eq. 3. $\Delta_{\eta r}$ is the UMTS load factor associated to a new call, given by the argument of the sum of Eq. 2, calculated with the appropriate parameters for each type of call [6]. $r$ is the WLAN mean bit rate requested by the new call.

\section{Simulation Scenario}

In order to demonstrate and evaluate the joint radio resource management strategy proposed in this paper, we considered a simulation scenario corresponding to an hotspot located in a shopping center. We adopted simulation scenarios where the number of users varies from 1 to 1000 and, in the busy hour, each user is involved, in average, in 6 calls, each with an average duration of $120 \mathrm{~s}$. The incoming calls follow a Poisson process with mean inter-arrival interval given by

$$
1 / \lambda=\frac{3600}{U_{q} \times U_{c}}
$$


where $U_{q}$ is the average number of users and $U_{c}$ is the average number of calls made by a user in the busy hour.

The simulation system was implemented in Network Simulator 3 (NS-3) [10], being the UMTS interface developed as a statistical module, based in [6], [11]. NS-3 already implements the IEEE 802.11 multi-rate standard (i.e., 6 Mbps to $54 \mathrm{Mbps}$ ) [12], [9]. Voice and videostreaming applications are modeled respectively as constant bit rate (CBR) and variable bit rate (VBR) traffic, transported using UDP. www and FTP are modeled using the On-Off traffic source implemented in NS-3. The www application alternates between the On and the Off states, while FTP is modeled as constantly On, considering files sufficiently large to occupy a 120s session. www and FTP traffic is transported using TCP. We assumed that the UMTS network supports applications with QoS requirements, while the WLAN network does not integrate any mechanism for QoS support.

In UMTS network, the usual value for the maximum load factor, $\eta_{\max }$, is 0.75 [6]. Fig. 3 shows the $\eta_{\max }$ distribution in the context of an hotspot scenario. We assumed that the user density inside the hotspot is, in average, twice the density in the remain of the UMTS cell. In this case, the load factor inside the intersection area of both technologies was considered 0.50 , being the remaining 0.25 applied to the outside of the intersection area.

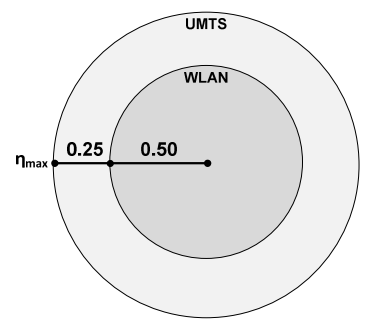

Fig. 3. $\eta_{\max }$ distribution according to users location - inside/outside hotspot

\section{RESUlts ANALYSiS}

In this section we present and discuss the results obtained through the simulation of three joint multi-radio resource management strategies using the scenario described in Section V. The objective is to compare the behavior of the strategy presented in this paper, identified as Mobility Tendency (MTend), with two other strategies, namely the Load Balancing (LBal) strategy and the strategy based in the Coverage Area (CovAr).

We separate the analysis in two different scenarios. The former scenario only considers real-time applications, concretely voice and videostreming, while the later scenario considers real-time and non-real-time applications running concurrently.

The parameter used to compare the three strategies is the call blocking probability (CBP). This probability is given by

$$
C B P(\%)=\left[1-\frac{C_{a c p t}}{C_{o f f}}\right] \times 100,
$$

where $C_{a c p t}$ is the number of application calls accepted, and $C_{\text {off }}$ is the total number of calls offered to the system.

\section{A. Real-Time Traffic}

To analyze the behavior of the three joint radio resource management strategies in face of real-time traffic, we considered scenarios using voice and videostreaming traffic parameterized according to the Table I [13], [14]. We assumed that $50 \%$ of the calls are voice calls and the other $50 \%$ are videostreaming calls, i.e., only the two first applications from Table II were considered in these simulations.

Fig. 4 (a) shows the average values and the $95 \%$ confidence intervals of the call blocking probability for all strategies. These strategies present very high probablities for a number of users above 600 , reaching a call blocking probability of almost $100 \%$ between 900 and 1000 users. The MTend strategy presents a performance better than the other two, having a call blocking probability always below the other two strategies until the number of users reaches 500 .

The box inside Fig. 4 (a) shows the performance of the resource management strategies for scenarios with a reduced number of users, from 1 to 40. The MTend strategy still has a better performance than the other two strategies in those scenarios, obtaining call blocking probabilities under $2 \%$ for a number of users less than 20 .

The most interesting analysis should be done for low call blocking probabilities, under $20 \%$, which are the values considered by an operator for a real service provisioning scenario. Fixing a call blocking probability, the gain obtained with the MTend strategy comparing with the concurrent strategy $S$ is given by Eq. (8), where $U \operatorname{ser}_{S}$ represents the number of users using the operator's networks with the strategy $S$.

$$
G_{M T e n d \_S}^{C B P}=\frac{U \operatorname{ser} s_{M T e n d}-U \operatorname{ser} s_{S}}{U \operatorname{ser} s_{M T e n d}}
$$

Considering the call renegotiation mechanism active, for example, for a call blocking probability of $2 \%$, the gain of $G_{M T e n d \_L B a l}^{2 \%}=6 \%$, while for the CovAr strategy, the gain is $G_{\text {MTend_CovAr }}^{2 \%}=35 \%$. It means that the operator supports $6 \%$ to $35 \%$ more users with the MTend strategy than with the other two resource management strategies, for the given call blocking probability.

Fig. 4 (b) shows the average values and the same confidence intervals for call blocking probability, now assuming that the three strategies integrate the renegotiation mechanism described in Section IV.

Comparing Fig. 4 (a) with 4 (b), becomes clear that the CovAr strategy increases its performance when it uses the renegotiation mechanism; this occurs because of the succeeded renegotiation of calls, mainly in scenarios where the number of users is low (between 100 and 400). Even though, the MTend strategy presents a performance better than the other two, until the number of users reaches 600 . This is due mainly to the use of the reallocation mechanism implemented in the MTend strategy, which takes advantage of the resources available at both interfaces. In this case, the reallocation of calls from the congestioned network to the other network occurs. Above the 600 users, the call inter-arrive interval becomes small and the reallocation mechanism acts more frequently to maintain 


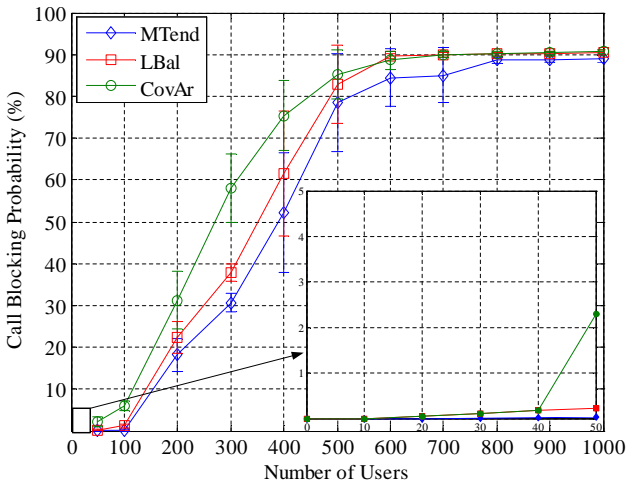

(a)

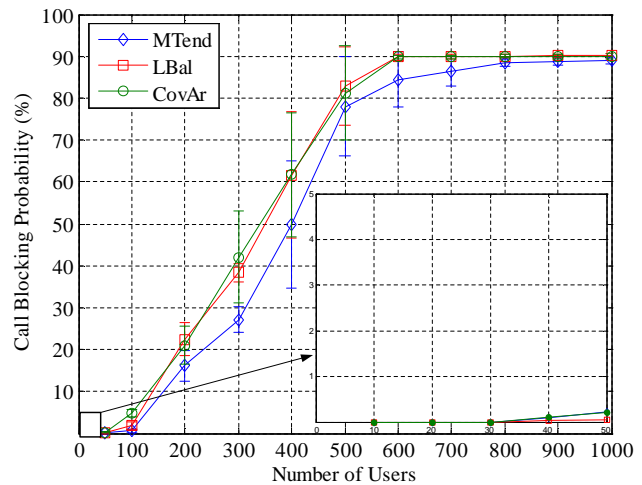

(b)

Fig. 4. Call blocking probability for real-time traffic, without (a) and with (b) call renegotiation

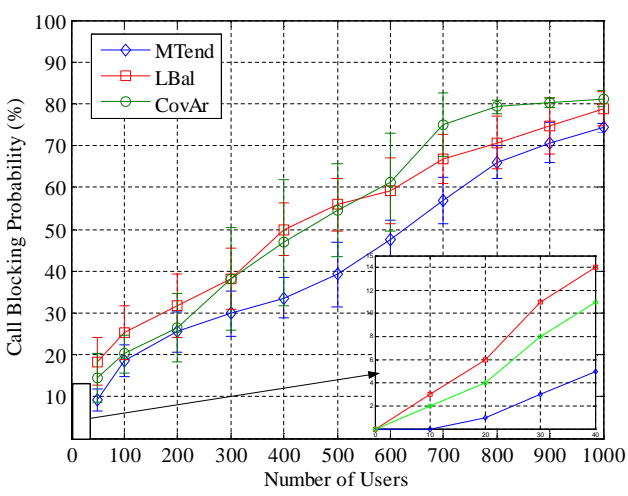

(a)

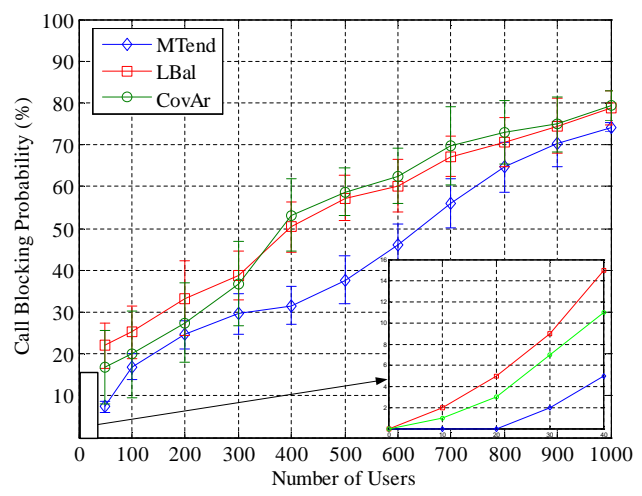

(b)

Fig. 5. Call blocking probability for real-time and non-real-time traffic, without (a) and with (b) call renegotiation

the networks balanced, what increases the number of blocked calls. Table III summarizes the gain obtained in real-time traffic scenarios with the MTend strategy in comparison with the other two strategies, considering call blocking probabilities varying from $2 \%$ to $10 \%$.

TABLE III

GAIN OF MTEND StRATEgy IN RESPECt TO LBAL AND COVAR FOR REAL-TIME TRAFFIC

\begin{tabular}{|c|c|c|c|c|}
\hline \multirow{2}{*}{ CBP(\%) } & \multicolumn{4}{|c|}{$G_{M T \text { ) }}^{C B P}$ ) } \\
\cline { 2 - 5 } & Without Renegotiation & With Renegotiation \\
\cline { 2 - 5 } & S=LBal & S=CovAr & S=LBal & S=CovAr \\
\hline 2 & 8 & 56 & 6 & 35 \\
\hline 5 & 12 & 33 & 7 & 26 \\
\hline 10 & 14 & 30 & 9 & 29 \\
\hline
\end{tabular}

\section{B. Real-Time and Non-Real-Time Traffic}

For the scenario with real-time and non-real-time traffic the simulations considered all the four applications presented in the Table I, where the average number of calls made by each user per hour is given in Table IV.

Fig. 5 shows the average values and the $95 \%$ confidence intervals of the call blocking probability for the three strategies obtained with the introduction of TCP traffic (www and FTP), in addiction to the real-time traffic (voice and videostreaming).
TABLE IV

CALLS PER USER PER HOUR IN REAL-TIME AND NON-REAL-TIME TRAFFIC SIMULATIONS

\begin{tabular}{l|l} 
Application & Average call per user per hour \\
\hline Voice & 2 \\
Videostreaming & 2 \\
Www & 1 \\
FTP & 1
\end{tabular}

In Fig. 5 (a) the strategies do not integrate the call renegotiation mechanism, while in Fig. 5 (b) this mechanism is integrated in all the three strategies.

Comparing Fig. 5 (a) with Fig. 5 (b), we conclude that the call renegotiation mechanism increases the performance of all the strategies, although not for the same number of users. However, the call renegotiation mechanism benefits are not so relevant as observed with real-time traffic, in Fig. 4, which means that with TCP traffic the call renegotiation mechanism were less used by the strategies.

Comparing Fig. 5 with Fig. 4, we should stress two aspects. The former has to do with the behavior of the call blocking probability when the number of users increases. In face of TCP traffic, the call blocking probability increases much more gradually than when only real-time traffic is present, where this probability increases rapidly between 100 and 500 users. The 
reason for the gradual increase of call blocking probability in the real-time and non-real-time traffic scenarios is related with the higher diversity of applications competing for the same resources. In this scenario the number of videostreaming calls decreases, which explains the lower call blocking probabilities, even for higher number of users.

The other relevant aspect is associated with the call blocking probability for a low number of users. Comparing the boxes inside Figs. 4 and 5, it can be observed that for the real-time and non-real-time traffic scenario the call blocking probability starts to grow right at the beginning of the graphic, while in real-time traffic scenario this probability remains constantly low until 50 users. However, the MTend strategy outperforms the other two strategies, even for this number of users.

Table V summarizes the gain obtained in real-time and non-real-time traffic scenarios with the MTend strategy in comparison with the other two strategies, considering call blocking probabilities varying from $2 \%$ to $10 \%$. By comparing Table V with Table III, we can observe that the gains presented by MTend strategy over the other strategies increase for the real-time and non-real-time traffic scenario; that is, the gains in the this scenario, which supports also www and FTP applications, are higher than in the real-time traffic scenario, where only voice and videostreaming applications were used.

TABLE V

GAIN OF MTEND STRATEGY IN RESPECT TO LBAL AND COVAR FOR REAL-TIME AND NON-REAL-TIME TRAFFIC

\begin{tabular}{|c|c|c|c|c|}
\hline \multirow{3}{*}{ CBP(\%) } & \multicolumn{4}{|c|}{$G_{\text {MTend_S }}^{C B P}(\%)$} \\
\cline { 2 - 5 } & Without Renegotiation & \multicolumn{2}{|c|}{ With Renegotiation } \\
\cline { 2 - 5 } & S=LBal & S=CovAr & S=LBal & S=CovAr \\
\hline 2 & 10 & 60 & 67 & 50 \\
\hline 5 & 22 & 45 & 50 & 38 \\
\hline 10 & 36 & 33 & 52 & 42 \\
\hline
\end{tabular}

An important aspect that contributed to the better performance of MTend strategy, compared with the other two strategies, is the different packet size of the simulated applications. Videostreaming, www and FTP applications have their packets larger than the voice application packets, which makes the WLAN medium access more efficient in terms of throughput. In [12], [15] the WLAN throughput performance is analyzed for several packet size scenarios. These studies show that the increase of throughput is proportional to the increase of packet size, what was also confirmed with our results. The MTend strategy takes also advantage of this fact.

\section{CONCLUSION}

This paper presents a performance analysis of three joint radio resource management strategies for locations simultaneously covered by UMTS and WLAN networks. This analysis compares the behavior of two well-known strategies in this context, one based on the coverage area and the other based on load balancing, with a new strategy proposed by the authors, the MTend strategy. This new strategy distributes efficiently the new calls by both network interfaces, using criteria related with the mobility of users. Besides, it also introduces mechanisms for call renegotiation and call reallocation, which showed to significantly increase the global system performance.

The simulation results presented in the paper show that the MTend strategy has a performance which is globally better than the two other strategies used for comparison, either in face of real-time traffic or in face of real-time and non-realtime traffic. Besides accepting more calls, the MTend strategy guaranties a better QoS support for the applications. This strategy tends also to decrease the number of handoffs between the UMTS and the WLAN interfaces, giving priority to voice calls in the UMTS interface.

For future work, we intend to step forward the present performance analysis, including also some economic parameters in the study, such as the total revenue and the revenue per interface for a given period of exploration time.

\section{REFERENCES}

[1] 3GPP, "3GPP System to Wireless Local Area Network (WLAN) Interworking," Services and System Aspects Group, TR-23.234, March 2007.

[2] — , "Mobility Between 3GPP-Wireless Local Area Network (WLAN) Interworking and 3GPP Systems (Release 8)," Services and System Aspects Group, TS-23.327 v.8.2.0, December 2008.

[3] R. Agustí, "Radio Resource Management in Beyond 3G Systems," in IEEE Mediterranean Electrotechnical Conference (MELECON 2006), 2006, pp. 569-574.

[4] S. Ramirez, M. Genoves, M. Navarro, R. Skehill, and S. McGrath, "Evaluation of Policy-Based Admission Control Algorithms for a Joint Radio Resource Management Environment," in IEEE Mediterranean Electrotechnical Conference (MELECON 2006), 2006, pp. 599-603.

[5] A. Tolli, P. Hakalin, and H. Holma, "Performance Evaluation of Common Radio Resource Management (CRRM)," in IEEE International Conference on Communications (ICC 2002), 2002, pp. 3429-3433.

[6] H. Holma and A. Toskala, WCDMA For UMTS - HSPA Evolution and LTE. John Wiley \& Sons, Ltd, 2007.

[7] ETSI, "Selection procedures for the choice of radio transmission technologies of the UMTS," TR-101.112 v.3.2.0, April 1998.

[8] A. Bazzi, M. Diolaiti, C. Gambetti, and G. Pasolini, "WLAN Call Admission Control Strategies for Voice Traffic over Integrated 3G/WLAN Networks," in 3rd IEEE Consumer Communications and Networking Conference, (CCNC 2006), vol. 2, 2006, pp. 1234-1238.

[9] H. Zhai, X. Chen, and Y. Fang, "How Well can the IEEE 802.11 Wireless LAN Support Quality of Service?" IEEE Transactions on Wireless Communications, vol. 4, no. 6, pp. 3084-3094, 2005.

[10] NS-3, "Network simulator 3, online: http://www.nsnam.org." [Online]. Available: http://www.nsnam.org

[11] J. Pérez-Romero, O. Sallent, and R. Agustí, Radio Resource Management Strategies in UMTS. John Wiley \& Sons, Ltd, 2005.

[12] G. Bianchi, "Performance Analysis of the IEEE 802.11 Distributed Coordination Function," IEEE Journal on Selected Areas in Communications, vol. 18, no. 3, pp. 535-547, March 2000.

[13] 3GPP, "Improvement of RRM across RNS and RNS/BSS (Release 5)," Radio Access Network Group, TR-25.881 v.5.0.0, December 2001.

[14] P. T.-G. D. Staehle, K. Leibnitz, "Source Traffic Modeling of Wireles Applications," University of Wurzburg, Research Report 261, June 2000.

[15] H. Chen and Y. Li, "Performance Model of IEEE 802.11 DCF with Variable Packet Length," IEEE Communications Letters, vol. 8, no. 3, pp. 186-188, March 2004. 